\title{
Total Hip Replacement in Developmental Hip Dysplasia: A Narrative Review
}

George C. Papachristou ${ }^{1}$, Eleni Pappa ${ }^{2}$, Dimitrios Chytas ${ }^{3}$, Panagiotis T. Masouros ${ }^{4}$, Vasileios S. Nikolaou $^{5}$

1. 2nd Department of Orthopaedics, School of Medicine. National and Kapodistrian University of Athens, Athens, GRC 2. 5th Department of Orthopaedics, "KAT" General Hospital of Athens, Athens, GRC 3. Department of Orthopaedics, European University of Cyprus, Nicosia, CYP 4. Department of Orthopaedics, General Hospital Evangelismos, Athens, GRC 5. 2nd Department of Orthopaedics, National and Kapodistrian University of Athens School of Medicine, Athens, GRC

Corresponding author: Vasileios S. Nikolaou, vassilios.nikolaou@gmail.com

\section{Abstract}

The reconstruction of the hip joint in patients suffering from developmental hip dysplasia (DDH) is a demanding procedure and presents many challenges to the reconstructive surgeon. Higher rates of mechanical complications are present in this group of patients. The results of cemented and uncemented implants used in DDH patients are very promising, according to recent outcomes. However, the surgeon has to be aware of several complications, in order to establish an uneventful surgical management of DDH. The specific article investigates the technical challenges and clinical results of total hip arthroplasty in patients with DDH.

Review began 04/20/2021 Review ended 04/29/2021 Published 04/29/2021

(c) Copyright 2021

Papachristou et al. This is an open access article distributed under the terms of the Creative Commons Attribution License CC-BY 4.0., which permits unrestricted use, distribution, and reproduction in any medium, provided the original author and source are credited.
Categories: Orthopedics

Keywords: developmental hip disease, total hip replacement, hip arthritis, total hip arthroplasty, congenital hip dysplasia

\section{Introduction And Background}

Developmental hip dysplasia (DDH) encompasses a spectrum of progressive changes to both the femur and the acetabulum, which eventually lead to secondary osteoarthritis of the hip joint. The abnormal bone morphology, growth and orientation, as well as soft tissue alterations, completely distort the normal relation between acetabulum and femoral head. Under these circumstances, THA in this population can be unpredictably challenging [1] with a reasonably high complication rate, compared to primary osteoarthritis. Aside from the morphological challenges, surgeons must also consider the usually younger age of the patients and the subsequent need for revision surgery [2]. An understanding of the common acetabular and femoral morphologic abnormalities will aid the surgeon in preparing for the complexity of the surgical case. In this article, we attempt a comprehensive review of the technical challenges and outcomes of THA in patients with DDH, including emphasis on the most suitable acetabular and femoral components and the potential reasons for postoperative complications and their management. A systematic search of the PubMed database was conducted using "dysplastic hip" and "total hip arthroplasty" as key phrases from 2000 to 2020. Studies involving pediatric patients were excluded (Figure 1). 


\section{Cureus}

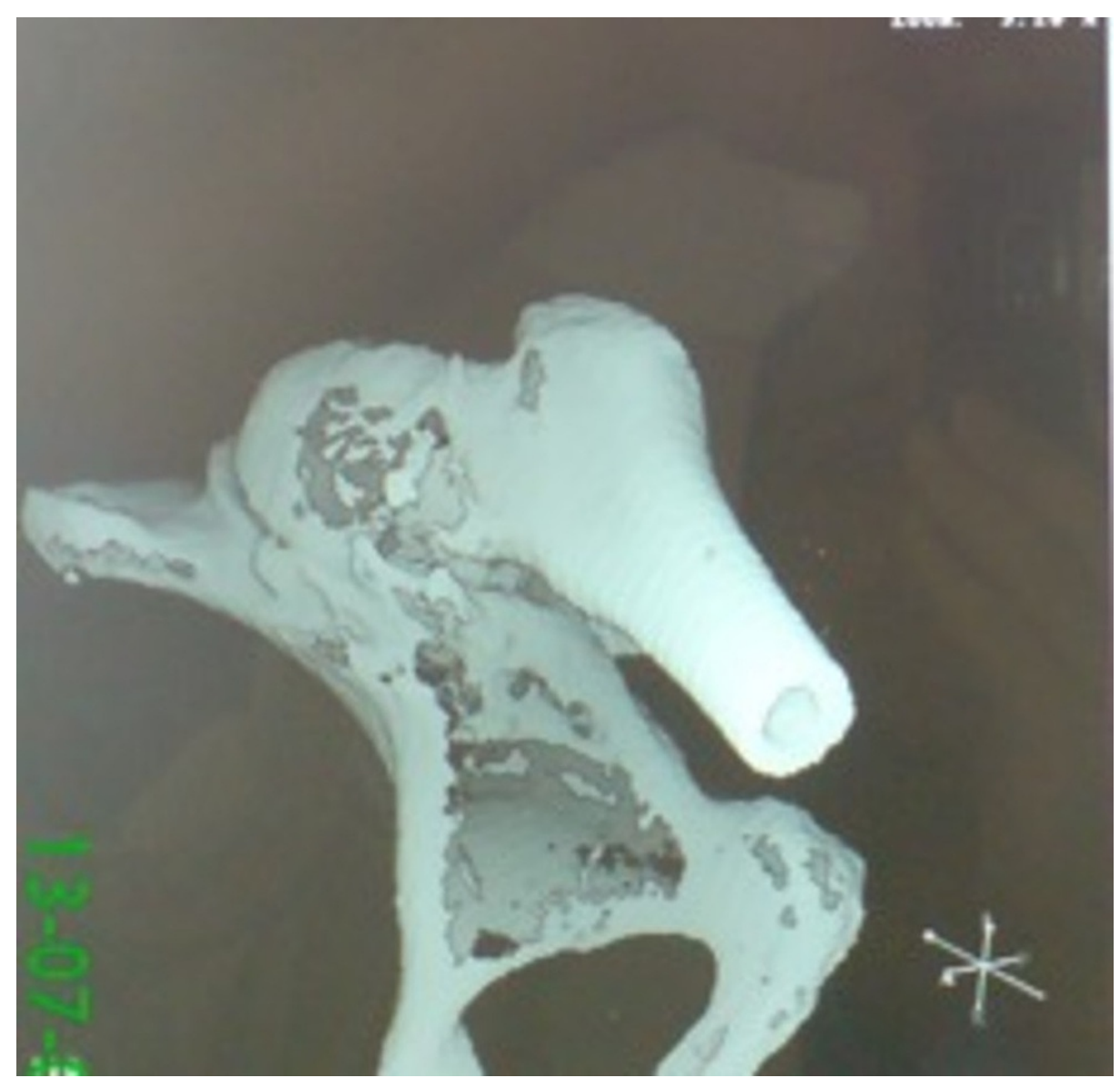

FIGURE 1: 3D hemipelvis remodelling from CT scan of dysplastic hip.

\section{Review}

\section{Classification}

The significant heterogeneity of dysplastic hips necessitates a classification system, so that investigators could use the same terminology and compare their results. One of the most widely used classifications, as proposed by Crowe et al. [3], is based on the extent of proximal displacement of the femoral head with respect to the vertical height of the pelvis. Alternatively, proximal migration can be expressed by dividing the distance between the inter-teardrop line and the femoral head-neck junction with the line connecting the ischial tuberosities and the line connecting the iliac crests, being characterized in the current literature as quantitative. Hartofilakidis et al. classification, which ensued, describes three distinct entities: mildly dysplastic, low and high dislocation, based on the anatomical abnormalities. In the case of the aforementioned classification, it has been mentioned in the current literature that it is lacking in providing information regarding the anatomical structural changes especially during hip surgery [4-6]. However, both classifications have been used for both clinical practice and research purposes.

Both classifications were evaluated as having substantial to almost perfect inter- and intraobserver agreement [7]. Kose et al. [8] found substantial inter- and intra-observer agreement for Crowe classification and substantial to moderate agreement for the Hartofilakidis one. We agree with Kose et al. [8], who concluded that each system has its own advantages and disadvantages, suggesting the need for both of these classifications together to increase accuracy. Nevertheless, we consider that Hartofilakidis et al classification is simpler than the one by Crowe et al., as it describes better the anatomical differences that the surgeon faces during the operation.

Recently Gaston et al. [9] described a new classification system for adult DDH with separate classifications for acetabulum and femur, whereas Clavé et al. $[10,11]$ underlined the significance of the preoperative planning of total hip replacement in DDH by using both classification systems (Tables 1, 2, 3). 


\title{
Cureus
}

\begin{tabular}{|c|c|}
\hline Type I & $<50 \%$ femoral head subluxation \\
\hline Type II & $50 \%-75 \%$ femoral head subluxation \\
\hline Type III & $75 \%-100 \%$ femoral head subluxation \\
\hline Type IV & $>100 \%$ femoral head subluxation \\
\hline
\end{tabular}

\section{TABLE 1: Classification system of congenital hip disease in adults according to Crowe et al.}

\begin{tabular}{|l|l|}
\hline Dysplastic hip & The femoral head is contained within the original acetabulum despite the degree of subluxation. \\
\hline Low dislocation & The femoral head articulates with a false acetabulum that partially covers the true acetabulum. \\
High dislocation & The femoral head is completely out of the true acetabulum and has migrated superiorly and posteriorly. \\
\hline
\end{tabular}

TABLE 2: Classification system of congenital hip disease in adults according to Hartofilakidis et al.

\begin{tabular}{|l|l|}
\hline Al & Dysplastic acetabulum \\
\hline All & The acetabulum associated with a low femoral dislocation \\
Allla & The post-surgical acetabulum: with retained metalwork \\
Alllb & The post-surgical acetabulum: without retained metalwork \\
\hline
\end{tabular}

TABLE 3: Classification system of congenital hip disease in adults according to Gaston et al.

\begin{abstract}
Surgical pearls and challenges
Elaborate preoperative planning and availability of a wide range of implants are imperative. The morphological changes, the location of the native acetabulum, the extent of the proximal displacement of the femoral head and the combined anteversion all need to be considered pre-operatively. Standing fulllength X-rays are used to assess leg-length discrepancy (LLD) and determination of the subtrochanteric osteotomy. The accurate size of the prosthesis as well as the specific site of the acetabular cup position should also be determined. Cups and heads of a smaller size need to be available on site. 3D preoperative planning can be a useful asset. Current literature suggests that CT-based three-dimensional templating provides the best accuracy in predicting the anatomic location of the acetabulum, but also the acetabular bone stock and bony landmarks. Fluoroscopy has also been used but the 2D images are considered to overestimate the present bone adequacy due to the bone shield.
\end{abstract}

In mildly dysplastic hips, standard approaches including anterior, anterolateral, and posterior can be utilized depending on surgeon's preferences. However, in cases of severe dysplasia a posterior approach is favored to allow for adequate exposure of the femoral head and acetabulum [12].

Reconstruction of the acetabular side is the most problematic part due to the deficient bone stock. Identification of the true acetabulum and re-establishment of the normal hip center are crucial, although not always feasible [13]. After resection of the femoral head, three steps can aid in locating the native acetabulum: (a) the ligamentum teres is followed distally to the cotyloid fossa, (b) the inferior capsule is identified, which is likely to be in line with the transverse ligament, (c) the bony landmarks (anterior and posterior). A triangle-shaped fossa, inferior to the pseudoacetabulum, which typically contains fatty tissue reveals the location of the true acetabulum. The native acetabulum has usually a small diameter requiring smaller components (usually 38 to $52 \mathrm{~mm}$ ), in comparison with primary osteoarthritis [14]. If the pseudoacetabulum is mistakenly identified as the native one, this can result in an oversized acetabular cup, placed in a high and lateral position [13].

Medialization of the anatomic hip joint, in order to accommodate the whole cup, can be achieved by means of either controlled reaming and deepening of the acetabulum or the so-called cotyloplasty (osteotomy of 
the medial acetabular wall and medial advancement) $[15,16]$. However, cotyloplasty has been associated with complications such as cup migration, loosening and osteolysis. Failure of the initial fixation and protrusion of the cup into the pelvis have also been reported. Moreover, revision after cotyloplasty, can be troublesome due to the thin medial wall.

The femur in DDH is generally characterized by increased neck-shaft angle, excessive anteversion and a relatively small diaphyseal diameter [13]. Interestingly, Sugano et al. [17] reported an anteversion of more than 40 degrees in $25 \%$ of their patients, while Noble et al. [18] also calculated a mean increase in femoral anteversion by 5 to 16 degrees. The anteversion can be adjusted precisely using either a modular stem or the modified S-ROM stem which is based on 3D reconstruction of proximal femur based on CT data. Regarding monoblock stems, the findings in the literature are debatable. On the one hand, these stems may not be suitable for severe DDH, on the grounds that stem anteversion after THA with a tapered wedge can be greater in comparison with a metaphyseal filling stem [19-21]. On the other hand, in the case of severe femoral distortions where no merchandise implants may be available, a customized femoral prosthesis is likely to be used based on the principles of computer-aided engineering.

Besides bony changes, soft tissue alterations should also be taken into account. The articular capsule is thickened and upwards elongated, while the function of the abductors is impaired. With the possible exception of the abductors, all the muscles around the hip joint are shortened in both low and high dislocations [22,23]. In most cases, brave intraoperative soft tissue release and tenotomies are advocated [24,25,26,27]. Wu et al. [28] described a soft-tissue release in four steps, starting with splitting of the adductor and parts of the iliotibial tract, followed by release of the iliopsoas tendon from the lesser trochanter, release of the attachments of the piriformis and hamstring muscles and finally osteotomy and advancement of the greater trochanter distally along with the gluteus medius insertion. Finally, the sciatic nerve is shortened, while the courses of the femoral nerve and the profunda femoris artery are distorted, thus putting them at increased risk during surgery.

\section{Outcomes}

Current evidence suggests that THA improves both the pain and the functional hip scores in patients with DDH. Inao and Matsuno [29] reported satisfactory mobility and improvement of pain after a mean follow-up of 12.9 years. Despite the overall functional improvement, patients suffering from severe DDH may continue to limp even post-operatively due to abductors' weakness.

Cemented acetabular reconstruction is falling out of favor due to the decreased survival rates. Despite the fact that cemented cups were combined with autogenous bone graft, the revision rate was satisfactory. However, these rates worsen dramatically due to graft collapse or socket loosening [11,14]. Uncemented acetabular components, with or without bone augmentation, are now widely used in mildly dysplastic hips, with considerably low revision rates in mid- and long-term follow-up. A Kaplan-Meier survivorship analysis predicted a rate of survival of the acetabular component of $96 \%$ at 15 years.

Concerning the use of the high hip center, the reports are limited in size. Perka et al. [26] stated that very good results were achieved in a relatively young patient population when the hip joint center had been properly restored, even if a small cup with a thin polyethylene liner was used. It is emphasized that the problems encountered during restoration of the center of rotation in THA for DDH correlated well with the severity of the dislocation and the clinical outcome was not found to be related with the severity of dislocation [30-33].

On the femoral side, the use of cement stems yield better outcomes compared to the acetabular side. However, the results are still inconsistent. In case of uncemented femoral components, the long-term results in DDH are lacking. The use of proximally fit uncemented components in hip dysplasia is challenging due to significant deformity, and often osteotomies and modular components are necessary to achieve an optimal fit [33]. Recently, Zhen et al [34] evaluated the results of uncemented press-fit Wagner femoral stems in patients with DDH in a mean follow-up of 7.7 years, where Harris hip score was improved, without any femoral fractures or stem revisions.

Femoral shortening via proximal femoral osteotomy and greater trochanter distal advancement can be associated with significant complications, while they allow for preservation of abductor mechanism as well as more flexibility in correction of rotational deformities. Patients with Crowe IV dysplastic hips treated with subtrochanteric osteotomy and uncemented components show generally excellent healing rates of the osteotomy, while a 10-year follow-up study also revealed improved lasting Harris hip scores in patients with dysplasia treated with modular femoral components and subtrochanteric osteotomy [34,35].

THA after previous joint preserving procedures, such as Salter, Chiari as well as the rotational acetabular osteotomy (RAO) or femoral osteotomies is considered as particularly demanding. However, Fukuri et al. reported similar functional results to patients who underwent THA in the presence of a rotational acetabular osteotomy [21]. Even in the case of the Bernese osteotomy, Parvizi et al. concluded that subsequent THA for DDH was not compromised [22]. On the other hand, poorer functional outcomes have been reported after 
combined periacetabular osteotomy (PO) and intertrochanteric valgus osteotomy [4]. On the femoral side, pre-existing osteotomies are associated with increased complications rate such as intraoperative fracture and nerve palsies. Though, the survivorship of THA after femoral osteotomies ranges from $43.7 \%$ to $100 \%$ [22].

\section{Complications}

The complications rate after THA in DDH patients is substantially higher when compared to THA due to primary osteoarthritis. They include fractures, dislocations, non-unions, loosening of the implants, neurovascular injuries, infections and impaired functional outcome.

Such complications come from the hypoplastic bones (deficient acetabulum and upper femur) and the anatomical alterations. Moreover, there are difficulties due to the hypoplastic bones and the necessity to insert especially designed implants for DDH cases.

Regarding sciatic nerve palsy, it is generally accepted that the extent of the lengthening should not exceed 3-4 cm. When osteotomy is applied intra-operatively, the sciatic nerve injury level ranges from $5 \%$ to $11.3 \%$. Eggli et al. [25] proposed that the main reason of the sciatic nerve injury is direct intra-operative damage rather than leg length discrepancy. Care should be taken intra-operatively by applying controlled tension and femur traction during reduction. Intra-op nerve monitoring has been also proposed for high riding DDH [36].

Intraoperative fractures are likely to occur in 5.2\%-26.8\% of THA in cases of DDH [37-40]. Otherwise, if the femoral canal is too narrow to allow the insertion of the smallest stem, splitting of the femur, as proposed by Li et al. [27], is an alternative. This is mainly due to the narrow femoral canal and can be successfully treated intra-operatively with wire-plate internal fixation [41,42].

LLD is another common complication. Gait asymmetry is likely to occur when LLD > 10 mm exists. Lumbar scoliosis, pelvic tilt and severe limp should be taken into consideration to prevent under-correction of limb length. In cases of severe leg length discrepancy, a subtrochanteric osteotomy can be performed [12]. Several techniques, including transverse, oblique, z-shaped, or the double chevron osteotomy, have been proposed $[23,24]$. Current evidence suggests that THA combined with sub-trochanteric osteotomy is likely to provide satisfactory functional results $[22,37,38,39]$. If clinically significant limping is mentioned post-operatively, physical therapy and a shoe lift should be considered, as well as re-operation. In cases of bilateral DDH, LLD should be addressed by THA of the contralateral hip .In cases of irreducible dysplastic hips with expected lengthening greater than $3 \mathrm{~cm}$, a two-stage procedure can be considered. In a case series of 17 patients with Crowe III and IV DDH, Yoon et al. concluded that two-stage THA using initial skeletal traction after extensive soft-tissue release, could provide an acceptable alternative solution with satisfying functional results [43].

Aseptic loosening is considered one of the leading causes for revision, especially in the younger populations due to the increased activity levels [34]. Li et al. [27] reported that the acetabular revision rates for aseptic loosening were $8 \%$ at five years and $26 \%$ at 10 years. Inao and Matsuno [28] reported aseptic loosening in three sockets (15\%) but not in the stems. The cumulative survival rate at 20 years was $78 \%$, with revision for loosening of the acetabular component as the end-point. However, new technologies regarding personalised and customized implants have also been used for DDH patients, as a specific guide using acetabular superolateral rim during THA for DDH is used to assist the production of an artificial acetabulum in adult DDH patients [37] . Wang et al studied the patient-specific instrument (PSI) in a study of 20 THA patients with DDH, leading to satisfying radiological and functional post-operative results (Figures 2, 3, 4) [43]. Additionally, the use of Metha short hip stem has drawn attention, as it is likely to bridge the gap between traditional straight design stems and hip resurfacing prostheses in THA in young DDH patients. The Metha short hip stem system is an uncemented neck-retaining monoblock or modular stem, which is made of a titanium alloy with a proximal rough titanium surface, with a proximal trapezoidal section allowing multipoint contact and providing 3-point fixation through the medial calcar region, the proximal lateral cortex and proximal posterior cortex $[44,45]$. 


\section{Cureus}

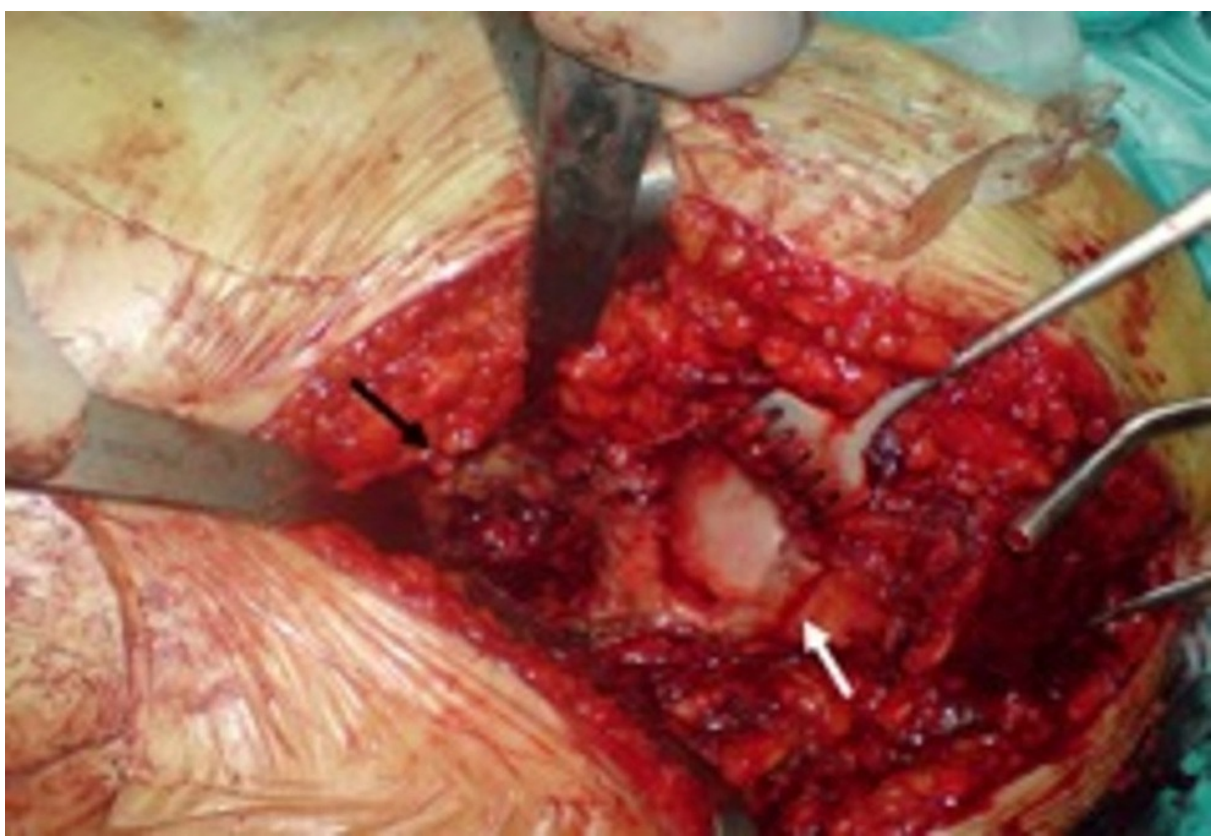

FIGURE 2: Intraoperative image of a patient with high dislocation.

The false acetabulum is indicated with the white arrow. The native, hypoplastic acetabulum is indicated with the black arrow. 


\section{Cureus}

\section{FIGURE 3: Postoperative image of the same patient.}

A trochanteric osteotomy was performed in order to facilitate the positioning of the hip joint to the lower native place. 


\section{Cureus}
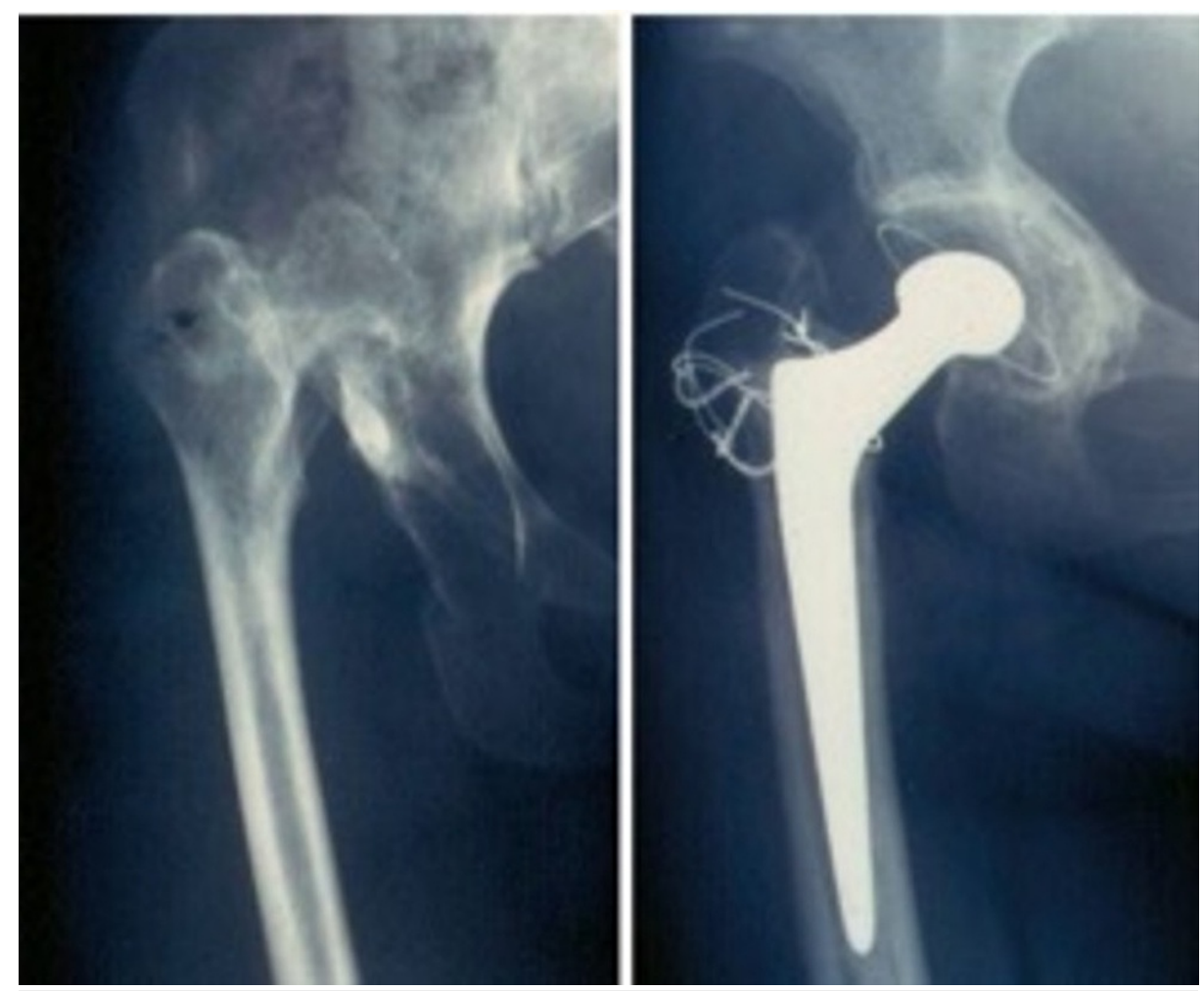

FIGURE 4: High CDH, before and after arthroplasty.

Cotyloplasty was necessary for the accommodation of the artificial cup. Nine years follow-up. Note the remodelling of the acetabulum roof.

\section{Conclusions}

Through a respectable number of publications over the last two decades, it seems that the challenges in DDH can be satisfyingly addressed using specifically designed implants, bone grafts and appropriate osteotomies. Placement of the cup in its anatomical location is a key factor, as it allows for restoration of the abductor muscles lever arm and normal hip center, thus providing superior outcomes in terms of biomechanics. Leg lengthening and equalization are crucial elements of the reconstruction. Historically, the outcomes of THA in DDH were reported to be poorer than those in hips without DDH. Nevertheless, current evidence suggests a trend of continuously improving outcomes in terms of pain relief, functional improvement, return to daily activities and reduction in complications rate. Unfortunately, there is a lack of high-quality studies to establish the optimal acetabular and femoral components. Future research should also focus on the impact of THA on the quality of life of this particular population.

\section{Additional Information}

\section{Disclosures}

Conflicts of interest: In compliance with the ICMJE uniform disclosure form, all authors declare the following: Payment/services info: All authors have declared that no financial support was received from any organization for the submitted work. Financial relationships: All authors have declared that they have no financial relationships at present or within the previous three years with any organizations that might have an interest in the submitted work. Other relationships: All authors have declared that there are no other relationships or activities that could appear to have influenced the submitted work.

\section{References}

1. Sanchez-Sotelo J, Trousdale RT, Berry DJ, Cabanela ME: Surgical treatment of developmental dysplasia of the hip in adults: I. Nonarthroplasty options. J Am Acad Orthop Surg. 2002, 10:321-33. 10.5435/00124635200209000-00004

2. Engesaeter LB, Furnes O, Havelin LI: Developmental dysplasia of the hip--good results of later total hip arthroplasty: 7135 primary total hip arthroplasties after developmental dysplasia of the hip compared with 59774 total hip arthroplasties in idiopathic coxarthrosis followed for 0 to 15 years in the Norwegian Arthroplasty Register. J Arthroplasty. 2008, 23:235-40. 10.1016/j.arth.2007.03.023

3. Crowe JF, Mani VJ, Ranawat CS: Total hip replacement in congenital dislocation and dysplasia of the hip . J Bone Joint Surg Am. 1979, 61:15-23. 
4. Osawa Y, Hasegawa Y, Okura T, Morita D, Ishiguro N: Total hip arthroplasty after periacetabular and intertrochanteric valgus osteotomy. J Arthroplasty. 2017, 32:857-61. 10.1016/j.arth.2016.08.023

5. Hartofilakidis G, Stamos K, Ioannidis TT: Low friction arthroplasty for old untreated congenital dislocation of the hip. J Bone Joint Surg Br. 1988, 70:182-6. 10.1302/0301-620X.70B2.3346284

6. Rogers BA, Garbedian S, Kuchinad RA, Backstein D, Safir O, Gross AE: Total hip arthroplasty for adult hip dysplasia. J Bone Joint Surg Am. 2012, 94:1809-21. 10.2106/JBJS.K.00779

7. Yiannakopoulos CK, Chougle A, Eskelinen A, Hodgkinson JP, Hartofilakidis G: Inter- and intra-observer variability of the Crowe and Hartofilakidis classification systems for congenital hip disease in adults. J Bone Joint Surg Br. 2008, 90:579-83. 10.1302/0301-620X.90B5.19724

8. Kose O, Celiktas M, Guler F, Baz AB, Togrul E, Akalin S: Inter- and intraobserver reliability of the Crowe and Hartofilakidis classifications in the assessment of developmental dysplasia of the hip in adult patients. Arch Orthop Trauma Surg. 2012, 132:1625-30. 10.1007/s00402-012-1600-x

9. Gaston MS, Gaston P, Donaldson P, Howie CR: A new classification system for the adult dysplastic hip requiring total hip arthroplasty: a reliability study. Hip Int. 2009, 19:96-101. 10.1177/112070000901900203

10. Clavé A, Kerboull L, Musset T, et al.: Comparison of the inter- and intra-observer reproducibility of the Crowe, Hartofilakidis and modified Cochin classification systems for the diagnosis of developmental dysplasia of the hip. Orthop Traumatol Surg Res. 2014, 100:S323-6. 10.1016/j.otsr.2014.07.007

11. Clavé A, Tristan L, Desseaux A, Gaucher F, Lefèvre C, Stindel E: Influence of experience on intra- and interobserver reproducibility of the Crowe, Hartofilakidis and modified Cochin classifications. Orthop Traumatol Surg Res. 2016, 102:155-9. 10.1016/j.otsr.2015.12.009

12. Macheras GA, Koutsostathis SD, Lepetsos P, Anastasopoulos PP, Galanakos S, Papadakis SA: THA following deformities due to congenital dislocation of the hip joint. Hip Int. 2014, 24:S29-32. 10.5301/hipint.5000168

13. Greber EM, Pelt CE, Gililland JM, Anderson MB, Erickson JA, Peters CL: Challenges in total hip arthroplasty in the setting of developmental dysplasia of the hip. J Arthroplasty. 2017, 32:S38-44. 10.1016/j.arth.2017.02.024

14. Hartofilakidis G, Babis GC, Lampropoulou-Adamidou K, Vlamis J: Results of total hip arthroplasty differ in subtypes of high dislocation. Clin Orthop Relat Res. 2013, 471:2972-9. 10.1007/s11999-013-2983-x

15. Kim M, Kadowaki T: High long-term survival of bulk femoral head autograft for acetabular reconstruction in cementless THA for developmental hip dysplasia. Clin Orthop Relat Res. 2010, 468:1611-20. 10.1007/s11999-010-1288-6

16. Chen M, Luo ZL, Wu KR, Zhang XQ, Ling XD, Shang XF: Cementless total hip arthroplasty with a high hip center for hartofilakidis type B developmental dysplasia of the hip: results of midterm follow-up. J Arthroplasty. 2016, 31:1027-34. 10.1016/j.arth.2015.11.009

17. Sugano N, Noble PC, Kamaric E, Salama JK, Ochi T, Tullos HS: The morphology of the femur in developmental dysplasia of the hip. J Bone Joint Surg Br. 1998, 80:711-9. 10.1302/0301-620x.80b4.8319

18. Noble PC, Kamaric E, Sugano N, Matsubara M, Harada Y, Ohzono K, Paravic V: Three-dimensional shape of the dysplastic femur: implications for THR. Clin Orthop Relat Res. 2003, 417:27-40.

19. Traina F, De Fine M, Tassinari E, Sudanese A, Calderoni PP, Toni A: Modular neck prostheses in DDH patients: 11-year results. J Orthop Sci. 2011, 16:14-20. 10.1007/s00776-010-0018-y

20. Fukui K, Kaneuji A, Sugimori T, Ichiseki T, Matsumoto T: Does rotational acetabular osteotomy affect subsequent total hip arthroplasty?. Arch Orthop Trauma Surg. 2015, 135:407-15. 10.1007/s00402-015-21545

21. Parvizi J, Burmeister H, Ganz R: Previous Bernese periacetabular osteotomy does not compromise the results of total hip arthroplasty. Clin Orthop Relat Res. 2004, 118-22. 10.1097/01.blo.0000128287.98083.63

22. Gallazzi E, Morelli I, Peretti G, Zagra L: What is the impact of a previous femoral osteotomy on THA? A systematic review. Clin Orthop Relat Res. 2019, 477:1176-87. 10.1097/CORR.0000000000000659

23. Papachristou G, Hatzigrigoris P, Panousis K, Plessas S, Sourlas J, Levidiotis C, Chronopoulos E: Total hip arthroplasty for developmental hip dysplasia. Int Orthop. 2006, 30:21-5. 10.1007/s00264-005-0027-1

24. Eggli S, Hankemayer S, Müller ME: Nerve palsy after leg lengthening in total replacement arthroplasty for developmental dysplasia of the hip. J Bone Joint Surg Br. 1999, 81:843-5. 10.1302/0301-620x.81b5.9610

25. Perka C, Fischer U, Taylor WR, Matziolis G: Developmental hip dysplasia treated with total hip arthroplasty with a straight stem and a threaded cup. J Bone Joint Surg Am. 2004, 86:312-9. 10.2106/00004623200402000-00014

26. Li X, Lu Y, Sun J, Lin X, Tang T: Treatment of Crowe type-IV hip dysplasia using cementless total hip arthroplasty and double chevron subtrochanteric shortening osteotomy: a 5- to 10-year follow-up study. J Arthroplasty. 2017, 32:475-9. 10.1016/j.arth.2016.07.050

27. Wu X, Li SH, Lou LM, Cai ZD: The techniques of soft tissue release and true socket reconstruction in total hip arthroplasty for patients with severe developmental dysplasia of the hip. Int Orthop. 2012, 36:1795-801. 10.1007/s00264-012-1622-6

28. Inao S, Matsuno T: Cemented total hip arthroplasty with autogenous acetabular bone grafting for hips with developmental dysplasia in adults: the results at a minimum of ten years. J Bone Joint Surg Br. 2000, 82:3757. 10.1302/0301-620x.82b3.10465

29. Zeng WN, Liu JL, Wang FY, et al.: Total hip arthroplasty for patients with Crowe type IV developmental dysplasia of the hip: ten years results. Int J Surg. 2017, 42:17-21. 10.1016/j.ijsu.2017.04.029

30. Rasi AM, Kazemian G, Khak M, Zarei R: Shortening subtrochanteric osteotomy and cup placement at true acetabulum in total hip arthroplasty of Crowe III-IV developmental dysplasia: results of midterm follow-up. Eur J Orthop Surg Traumatol. 2018, 28:923-30. 10.1007/s00590-017-2076-8

31. Kim M, Kadowaki T: High long-term survival of bulk femoral head autograft for acetabular reconstruction in cementless THA for developmental hip dysplasia. Clin Orthop Relat Res. 2010, 468:1611-20. 10.1007/s11999-010-1288-6

32. Hitz OF, Flecher X, Parratte S, Ollivier M, Argenson JN: Minimum 10-year outcome of one-stage total hip arthroplasty without subtrochanteric osteotomy using a cementless custom stem for Crowe III and IV hip dislocation. J Arthroplasty. 2018, 33:2197-202. 10.1016/j.arth.2018.02.055

33. Zhen P, Liu J, Lu H, Chen H, Li X, Zhou S: Developmental hip dysplasia treated by total hip arthroplasty 
using a cementless Wagner cone stem in young adult patients with a small physique. BMC Musculoskelet Disord. 2017, 18:192. 10.1186/s12891-017-1554-9

34. Rollo G, Solarino G, Vicenti G, Picca G, Carrozzo M, Moretti B: Subtrochanteric femoral shortening osteotomy combined with cementless total hip replacement for Crowe type IV developmental dysplasia: a retrospective study. J Orthop Traumatol. 2017, 18:407-13. 10.1007/s10195-017-0466-7

35. Imam MA, Fathalla I, Holton J, Nabil M, Kashif F: Cementless total hip replacement for the management of severe developmental dysplasia of the hip in the middle eastern population: a prospective analysis. Front Surg. 2016, 3:31. 10.3389/fsurg.2016.00031

36. Kong X, Chai W, Chen J, Yan C, Shi L, Wang Y: Intraoperative monitoring of the femoral and sciatic nerves in total hip arthroplasty with high-riding developmental dysplasia. Bone Joint J. 2019, 101-B:1438-46. 10.1302/0301-620X.101B11.BJJ-2019-0341.R2

37. Zhang H, Zhou J, Guan J, Ding H, Wang Z, Dong Q: How to restore rotation center in total hip arthroplasty for developmental dysplasia of the hip by recognizing the pathomorphology of acetabulum and Harris fossa?. J Orthop Surg Res. 2019, 14:339. 10.1186/s13018-019-1373-9

38. Shi XT, Cheng CM, Feng CY, Li CF, Li SX, Liu JG: Crowe type IV hip dysplasia treated by THA combined with osteotomy to balance functional leg length discrepancy: a prospective observational study. Orthop Surg. 2020, 12:533-42. 10.1111/os.12655

39. Shi XT, Li CF, Cheng CM, Feng CY, Li SX, Liu JG: Preoperative planning for total hip arthroplasty for neglected developmental dysplasia of the hip. Orthop Surg. 2019, 11:348-55. 10.1111/os.12472

40. Liu Z, Bell CD, Ong AC, Wu S, Li Z, Zhang Y: Direct anterior approach total hip arthroplasty for Crowe III and IV dysplasia. Arthroplast Today. 2020, 6:251-6. 10.1016/j.artd.2020.02.008

41. Kim YL, Nam KW, Yoo JJ, Kim YM, Kim HJ: Cotyloplasty in cementless total hip arthroplasty for an insufficient acetabulum. Clin Orthop Surg. 2010, 2:148-53. 10.4055/cios.2010.2.3.148

42. Yoon PW, Kim JI, Kim DO, Yu CH, Yoo JJ, Kim HJ, Yoon KS: Cementless total hip arthroplasty for patients with Crowe type III or IV developmental dysplasia of the hip: two-stage total hip arthroplasty following skeletal traction after soft tissue release for irreducible hips. Clin Orthop Surg. 2013, 5:167-73. 10.4055/cios.2013.5.3.167

43. Wang C, Xiao H, Yang W, Wang L, Hu Y, Liu H, Zhong D: Accuracy and practicability of a patient-specific guide using acetabular superolateral rim during THA in Crowe II/III DDH patients: a retrospective study. J Orthop Surg Res. 2019, 14:19. 10.1186/s13018-018-1029-1

44. Malahias MA, Tejaswi P, Chytas D, Kadu V, Karanikas D, Thorey F: The clinical outcome of the Metha short hip stem: a systematic scoping review. Hip Int. 2021, 31:24-33. 10.1177/1120700020903719

45. Benjamin B, Haddad FS: Management of limb length problems during total hip arthroplasty for patients with developmental dysplasia of the hip. Br J Hosp Med. 2020, 81:1-7. 10.12968/hmed.2019.0362 